\title{
ON THE RADIAL OSCILLATIONS OF A SPHERICAL THIN SHELL IN THE FINITE ELASTICITY THEORY*
}

\author{
By C.-C. WANG (The Johns Hopkins University)
}

1. Introduction. The radial oscillations of a circular cylinder for isotropic incompressible elastic materials were discussed by Knowles** [1, 2], Truesdell and Noll [3]. This paper concerns a similar problem for a spherical shell. While the previous authors attained exact solutions for both the general case of a shell with arbitrary thickness and the limiting cases of a thin shell, we shall consider the thin shell case only.

It is known that these oscillatory motions belong to certain special cases of the quasi-equilibrated motions found by Truesdell [4]. We shall reproduce part of his results in the next section.

Notations. We use spherical coordinated for both the undeformed and the deformed state of the shell. As usual we distinguish them by using majuscules and minuscules respectively.

Constitutive equation. We assume that the material is isotropic, incompressible, and elastic. It is known that the most general representation*** of the constitutive equation for this kind of material is

$$
\mathbf{T}=-p \mathbf{I}+f \mathbf{B}+g \mathbf{B}^{-1},
$$

where $\mathbf{T}$ is the stress tensor, $p$ is the undetermined hydrostatic pressure, $\mathbf{I}$ is the identity tensor, B is the left Cauchy-Green tensor of the deformation with respect to some fixed undistorted reference configuration and $f, g$, are functions of the principal invariants of $\mathbf{B}$. Since the material is incompressible, only density-preserving motions are possible. If we pick the undeformed state to be the reference configuration, the determinant of $\mathbf{B}$ has the value 1.

2. Quasi-equilibrated motions of a spherical shell. The general solution of the equations of motion as shown in [4] is

$$
r^{3}= \pm R^{3}+A, \theta= \pm \Theta+B, \varphi=\Phi+C,
$$

where $A=A(t)$ is an arbitrary function of time, and $B$ and $C$ are constants. The acceleration potential is

$$
-\zeta=\frac{1}{3 r}\left(-A^{\prime \prime}+\frac{A^{\prime 2}}{6 r^{3}}\right)
$$

For inflation of a spherical shell we take the positive sign in (2). For radial oscillations set $B=C=0$, whence (3) becomes

$$
-\zeta=-\left(r r^{\prime \prime}+\frac{3}{2} r^{2}\right)
$$

*Received August 3, 1964; revised manuscript received November 24, 1964.

** Knowles [2] remarked that the sperical case can be treated similarly to the cylindrical case.

***If a stored energy function is assumed, as in [1], [2], certain compatibility relations of the functions $f$ and $g$ must be satisfied. This assumption is not necessary for the following analysis. Except for minimum smoothness requirements, $f$ and $g$ are arbitrary. 
The stress tensor in this case was shown in [4] to be

$$
\mathbf{T}=-\rho \zeta \mathbf{I}+\mathbf{T}_{0},
$$

where $\rho$ is the density and $T_{0}$ is the general solution of the stress system corresponding to an equilibrium configuration. Therefore, substituting (2) into (1) and (5) we get

$$
T\langle r r\rangle=-\rho\left(r r^{\prime \prime}+\frac{3}{2} r^{\prime 2}\right)+\psi(t)+2 \int_{c}^{r}\left[\left(\frac{r^{2}}{R^{2}}-\frac{R^{4}}{r^{4}}\right) f-\left(\frac{r^{4}}{R^{4}}-\frac{R^{2}}{r^{2}}\right) g\right] \frac{d r}{r},
$$

where $T\langle r r\rangle$ is the physical component of the stress tensor, $\psi(t)$ is an arbitrary function of time and $c$ is an arbitrary positive number. The arguments of $f$ and $g$ are

$$
\begin{aligned}
\mathrm{I} & =\text { first invariant of } \mathrm{B}=\frac{R^{4}}{r^{4}}+\frac{2 r^{2}}{R^{2}}, \\
\mathrm{II} & =\text { second invar.ant of } \mathrm{B}=\frac{r^{4}}{R^{4}}+\frac{2 R^{2}}{r^{2}} .
\end{aligned}
$$

Since the motion is density-preserving, the third invariant of $\mathbf{B}$ has the value 1 .

3. Thin shell approximation. Denoting the ratios of the thicknesses of the deformed and the undeformed shell to the corresponding inner radii $r$ and $R$ by $\delta$ and $\Delta$ respectively, by (2) we have

$$
r^{3}=R^{3}+A, r^{3}(1+\delta)^{3}=R^{3}(1+\Delta)^{3}+A .
$$

If we neglect terms of second or higher order in delta, (8) reduces to

$$
r^{3} \delta=R^{3} \Delta \text {. }
$$

Differentiate (9) twice we get

$$
\delta^{\prime}=-3 \frac{r^{\prime}}{r} \delta, \quad \delta^{\prime \prime}=12\left(\frac{r^{\prime}}{r}\right)^{2} \delta-3 \frac{r^{\prime \prime}}{r} .
$$

Let the difference of the pressures on the inner and outer surfaces of the shell be $Q(t)$. Then by (6)

$$
\begin{aligned}
Q(t)=\rho\left[2 r r^{\prime \prime} \delta+2 r r^{\prime} \delta^{\prime}+r^{2} \delta^{\prime \prime}\right. & \left.+3 r^{\prime}\left(r^{\prime} \delta+r \delta^{\prime}\right)\right] \\
& -2 \int_{r}^{r(1+\delta)}\left[\left(\frac{r^{2}}{R^{2}}-\frac{R^{4}}{r^{4}}\right) f-\left(\frac{r^{4}}{R^{4}}-\frac{R^{2}}{r^{2}}\right) g\right] \frac{d r}{r} .
\end{aligned}
$$

Assuming that the response functions $f, g$ are continuous in $r$, and using (9) and (10) we get

$$
Q(t)=-\left\{\frac{\rho r^{\prime \prime}}{r^{2}}+\frac{2}{r^{3}}\left[\left(\frac{r^{2}}{R^{2}}-\frac{R^{4}}{r^{4}}\right) f-\left(\frac{r^{4}}{R^{4}}-\frac{R^{2}}{r^{2}}\right) g\right]\right\} R^{3} \Delta .
$$

This is the equation of motion of the thin shell.

4. Free oscillations. For free oscillation we have $Q(t)=0$, hence by (12)

$$
\rho r^{\prime \prime}+\frac{2}{r}\left[\left(\frac{r^{2}}{R^{2}}-\frac{R^{4}}{r^{4}}\right) f-\left(\frac{r^{4}}{R^{4}}-\frac{R^{2}}{r^{2}}\right) g\right]=0 .
$$


This equation can be integrated at once to yield the following energy equation:

$$
\frac{1}{2} \rho r^{\prime 2}+2 \int_{R}^{r}\left[\left(\frac{r^{2}}{R^{2}}-\frac{R^{4}}{r^{4}}\right) f-\left(\frac{r^{4}}{R^{4}}-\frac{R^{2}}{r^{2}}\right) g\right] \frac{d r}{r}=\frac{1}{2} \rho \xi^{2},
$$

where $\xi$ is the radial velocity of the inner surface at the undeformed state. Therefore oscillating motion is possible if

$$
r^{\prime \prime}(R-r)>0,
$$

and if the equation

$$
2 \int_{R}^{r}\left[\left(\frac{r^{2}}{R^{2}}-\frac{R^{4}}{r^{4}}\right) f-\left(\frac{r^{4}}{R^{4}}-\frac{R^{2}}{r^{2}}\right) g\right] \frac{d r}{r}=\frac{1}{2} \rho \xi^{2}
$$

has two distinct roots, one on each side of the neutral position $r=R$. In this case the roots $a>R$ and $b<R$ of (16) are the maximum and the minimum radii of the inner surface in the oscillation. The period of oscillation $\tau$ can then be obtained by integrating along the closed curve on the hodograph plane, i.e.,

$$
\tau=2 \int_{b}^{a} \frac{d r}{r^{\prime}}
$$

where $r^{\prime}$ can be obtained from (14).

We remark here that for sufficiently small $\xi$, condition (16) is a consequence of condition (15). Experimental results [5] seem to support the following restrictions on the response functions:

$$
f>0, \quad g \leq 0 .
$$

Then it is readily seen that condition (15) is satisfied. Furthermore, if $f$ stays away from zero by at least a certain positive constant $\epsilon$ for any deformation, then every given $\xi$ shall yield an oscillation.

5. Oscillation due to pressure impulse. Assuming that the shell suffers an impulsive pressure at $t=0$, i.e.,

$$
Q(t)= \begin{cases}0 & t \leq 0 \\ Q & t>0\end{cases}
$$

where $Q$ is a constant. The equation of motion becomes

$$
-Q \frac{r^{2}}{R^{3} \Delta}=\rho r^{\prime \prime}+\frac{2}{r}\left[\left(\frac{r^{2}}{R^{2}}-\frac{R^{4}}{r^{4}}\right) f-\left(\frac{r^{4}}{R^{4}}-\frac{R^{2}}{r^{2}}\right) g\right], \quad t>0 .
$$

The initial conditions are

$$
r=R, \quad r^{\prime}=0 \quad \text { at } \quad t=0 .
$$

We can integrate (23) and get the following energy equation:

$$
\frac{Q}{3 \Delta}\left(1-\frac{r^{3}}{R^{3}}\right)=\frac{1}{2} \rho r^{2}+\int_{R}^{r} \frac{2}{r}\left[\left(\frac{r^{2}}{R^{2}}-\frac{R^{4}}{r^{4}}\right) f-\left(\frac{r^{4}}{R^{4}}-\frac{R^{2}}{r^{2}}\right)\right] d r,
$$

From (20) we see that $r^{\prime \prime}(0)<0(>0)$ according to $Q>0(<0)$. Therefore, oscillating motion is possible if the equation 


$$
\frac{Q}{3},\left(1-\frac{r^{3}}{R^{3}}\right)=\int_{R}^{r} \frac{2}{r}\left[\left(\frac{r^{2}}{R^{2}}-\frac{R^{4}}{r^{4}}\right) f-\left(\frac{r^{4}}{R^{4}}-\frac{R^{2}}{r^{2}}\right) g\right] d r
$$

has a positive root $a>R(<R)$ according to $Q>0(<0)$, and if the acceleration $r^{\prime \prime}$ changes sign between $r=R$ and $r=a$. In this case the period of oscillation is

$$
\tau=2\left|\int_{R}^{a} \frac{d r}{r^{\prime}}\right|
$$

where $r^{\prime}$ can be obtained from (22). The neutral position is the root of

$$
-Q \frac{r^{2}}{R^{3} \Delta}=\frac{2}{r}\left[\left(\frac{r^{2}}{R^{2}}-\frac{R^{4}}{r^{4}}\right) f-\left(\frac{r^{4}}{R^{4}}-\frac{R^{2}}{r^{2}}\right) g\right],
$$

where $r^{\prime \prime}$ changes its sign.

6. Oscillations of a sealed shell. Suppose that the inner and outer pressure of the shell in the neutral position $r=R$ is a constant $P_{0}$. Let the outer pressure remain unchanged when the shell is set to oscillate by some initial radial velocity $\xi$, while the inner pressure varies according to some ideal gas law, say, $P V^{\gamma}=$ constant. Thus the inner pressure at the deformed state is

$$
P=P_{0}\left(\frac{R}{r}\right)^{3 \gamma},
$$

hence

$$
Q(t)=P_{0}-P=P_{0}\left[1-\left(\frac{R}{r}\right)^{3 \gamma}\right] .
$$

The equation of motion becomes

$$
-\frac{p_{0}}{R^{3} \Delta} r^{2}\left[1-\left(\frac{R}{r}\right)^{3 r}\right]=\rho r^{\prime \prime}+\frac{2}{r}\left[\left(\frac{r^{2}}{R^{2}}-\frac{R^{4}}{r^{4}}\right) f-\left(\frac{r^{4}}{R^{4}}-\frac{R^{2}}{r^{2}}\right) g\right] .
$$

The initial conditions are

$$
r=R, \quad r^{\prime}=\xi \text { at } t=0 .
$$

Integrating (28) we get ${ }^{1}$

$$
\begin{aligned}
\frac{P_{0}}{3 \Delta}\left\{\left[1-\left(\frac{r}{R}\right)^{3}\right]+\right. & \left.\frac{1}{\gamma-1}\left[1-\left(\frac{R}{r}\right)^{3(\gamma-1)}\right]\right\} \\
& =\frac{1}{2} \rho r^{\prime 2}-\frac{1}{2} \rho \xi^{2}+\int_{R}^{r} \frac{2}{r}\left[\left(\frac{r^{2}}{R^{2}}-\frac{R^{4}}{r^{4}}\right) f-\left(\frac{r^{4}}{R^{4}}-\frac{R^{2}}{r^{2}}\right) g\right] d r .
\end{aligned}
$$

For adiabatic processes it is known that $1<\gamma<5 / 3$. In this range, it is easily shown that the first term on the right hand side of (30) is always positive except at the neutral position $r=R$. Hence if (15) and (16) hold, then (30) yields an oscillation. Furthermore, if $a>R$ and $b<R$ are the roots of (16), then the corresponding roots $a_{1}>R$ and $b_{1}<R$ of (30) for $r^{\prime}=0$ lie between $a$ and $b$, i.e.,

$$
b<b_{1}<R<a_{1}<a .
$$

${ }^{1}$ If $\gamma=1$, the second term on the left hand side of (30) becomes $3 \log r / R$. 
The period of oscillation is

$$
\tau=2 \int_{b_{2}}^{a_{2}} \frac{d r}{r^{\prime}}
$$

where $r^{\prime}$ can be obtained from (30).

Acknowledgement. The author is indebted to Professor C. Truesdell for many critical remarks of this paper. His thanks are due to Miss Sara Turnbull for her assistance in preparing the manuscript for publication. The reseach here reported was carried out under a grant of the U. S. National Science Foundation to the Johns Hopkins University.

\section{REFERENCES}

1. J. A. Knowles, Q. Appl. Math. 18, 71-77 (1960)

2. J. A. Knowles, J. Appl. Math. 29, 283-286 (1962)

3. C. Truesdell and W. Noll, The non-linear field theories of mechanics (to appear as Handbuch der Physik Band III/3)

4. C. Truesdell, Arch. Rational Mech. Anal. 11, 106-113 (1962); Addendum 12, 427 (1963)

5. C. Truesdell and R. A. Toupin, Arch. Rational Mech. Anal. 12, 1-33 (1963) 